\title{
Adaptive Control of Electromagnetic Suspension System by HOPF Bifurcation
}

\author{
Aming Hao, Xiaolong Li, and Longhua She \\ College of Mechatronics Engineering and Automation, National University of Defense Technology, 109 Deya Road, \\ Changsha 410073, China \\ Correspondence should be addressed to Aming Hao; hao.aming@gmail.com
}

Received 9 June 2013; Revised 22 September 2013; Accepted 23 September 2013

Academic Editor: Ion Zaballa

Copyright (C) 2013 Aming Hao et al. This is an open access article distributed under the Creative Commons Attribution License, which permits unrestricted use, distribution, and reproduction in any medium, provided the original work is properly cited.

EMS-type maglev system is essentially nonlinear and unstable. It is complicated to design a stable controller for maglev system which is under large-scale disturbance and parameter variance. Theory analysis expresses that this phenomenon corresponds to a HOPF bifurcation in mathematical model. An adaptive control law which adjusts the PID control parameters is given in this paper according to HOPF bifurcation theory. Through identification of the levitated mass, the controller adjusts the feedback coefficient to make the system far from the HOPF bifurcation point and maintain the stability of the maglev system. Simulation result indicates that adjusting proportion gain parameter using this method can extend the state stability range of maglev system and avoid the self-excited vibration efficiently.

\section{Introduction}

With the success of the Shanghai Pudong International Airport link in China, magnetic levitation vehicle (maglev vehicle) through electromagnetic suspension (EMS) technology has come into our life $[1,2]$. The EMS provides nonmechanical contacting suspension force by means of electromagnets with an air gap controller realized through position, velocity, and acceleration feedback. Classical control theories have provided much of the design rules for many EMS including maglev vehicle [3,4] and magnetic suspension and balance system [5]. However, because the constant linear control strategies deteriorate rapidly with increasing deviations from the nominal operating point caused by significant changes in the suspended load, some approaches to the problem of ensuring consistent performance while operating point changes have been reported. A model reference adaptive controller to compensate for payload variations and external force disturbances has been given [6]. A gain scheduling method has been designed [7], where the nonlinear force/current/air-gap relationship of the magnetic suspension is successively linearized at various operating points with a suitable controller is designed for each of these operating points. Some other kinds of method also have been developed by [8-11].
However, the above papers have not discussed how to deal with this case in which the mass levitated changes abruptly. Because the EMS is inherently unstable, nonlinear dynamic system, the above methods may not guarantee its stability with abrupt changing mass levitated sometimes. When the EMS becomes unstable by an abrupt disturbance, it may produce vibration firstly corresponding to HOPF bifurcation of a nonlinear state space equation [12]. That is to say, when designing the EMS controller parameters, if we consider an additional constraint which makes the controller parameters always far away from the HOPF bifurcation points while the mass levitated varies, a larger state stable range will be attained. Then, the anti-abrupt-disturbance ability of the system will be enhanced. This paper studies how to design parameter self-tuning adaptive controller according to the above principle.

\section{One Degree of Freedom EMS Model}

The one degree of freedom EMS is shown schematically in Figure 1. It is composed of an electromagnet, an air gap sensor, a DSP controller, a current driver, and a levitated object. Through proper control algorithm in DSP, the object will be levitated stably with certain gap. 
Set the levitation air gap with the direction down as $s$, the expected air gap is $s_{0}$, the velocity of air gap changing is $y$, the gravity acceleration is $g$, the mass of the object levitated is $m$, the current in electromagnet is $I$, the resistance of the electromagnet is $R$, and the electromagnet voltage on the system equilibrium point is $U_{0}$.

Set the turns of the electromagnet coil as $N$, pole area of the electromagnet is $A$, and permeability of vacuum is $\mu_{0}$. Then, set $k=\left(\mu_{0} N^{2} A\right) / 4$ as the constant of electromagnet determined by $N, A$, and $\mu_{0}$.

Suppose that the following dual-loop controller acts on the system:

$$
U_{k}=K_{P}\left(c-c_{0}\right)+K_{D} \dot{c}+K_{I} I,
$$

where $K_{P}$ and $K_{D}$ are adjustable gap loop feedback parameters. $K_{P}$ is the proportional feedback coefficient, and $K_{D}$ is the differential feedback coefficient.

$K_{I}$ is the current loop feedback coefficient. By adopting the proper current feedback, the time constant of electromagnet can be greatly reduced. So in the band of Ems system, the transfer function from voltage to the current of electromagnet can be seen as proportional component and the controller design becomes more convenient [4].

Then the the system model can be written as follows [13]:

$$
\begin{gathered}
\dot{c}=y, \\
\dot{y}=g-\frac{k I^{2}}{m c^{2}}, \\
\dot{I}=-\left(\frac{c}{2 k} R-\frac{y}{c}\right) I+\frac{c}{2 k}\left[U_{0}+K_{I} I+K_{P}\left(c-c_{0}\right)+K_{D} y\right] .
\end{gathered}
$$

\section{System Analysis}

Setting $\dot{s}=0, \dot{y}=0$, and $\dot{I}=0$, the equilibrium point of the state variables can be obtained:

$$
s_{0}=I_{0} \sqrt{\frac{k}{m g}}, \quad y_{0}=0, \quad I_{0}=\frac{U_{0}}{R-K_{I}} .
$$

Set $R^{\prime}=R-K_{I}$. is

The Jacobian matrix of the system at the equilibrium point

$$
A\left(K_{P}, K_{D}\right)=\left[\begin{array}{ccc}
0 & 1 & 0 \\
\frac{2 g}{s_{0}} & 0 & -\frac{2}{s_{0}} \sqrt{\frac{k g}{m}} \\
\frac{K_{P} s_{0}}{2 k} & \frac{s_{0} K_{D}}{2 k}+\sqrt{\frac{m g}{k}} & -\frac{R^{\prime} s_{0}}{2 k}
\end{array}\right] .
$$

The eigen polynomial of the Jacobian matrix of the system at the equilibrium point is

$$
\begin{aligned}
|\lambda I-A|= & \lambda^{3}+\frac{R^{\prime} s_{0}}{2 k} \lambda^{2}+K_{D} \sqrt{\frac{g}{k m}} \lambda \\
& +K_{P} \sqrt{\frac{g}{k m}}-\frac{g}{k} R^{\prime} .
\end{aligned}
$$

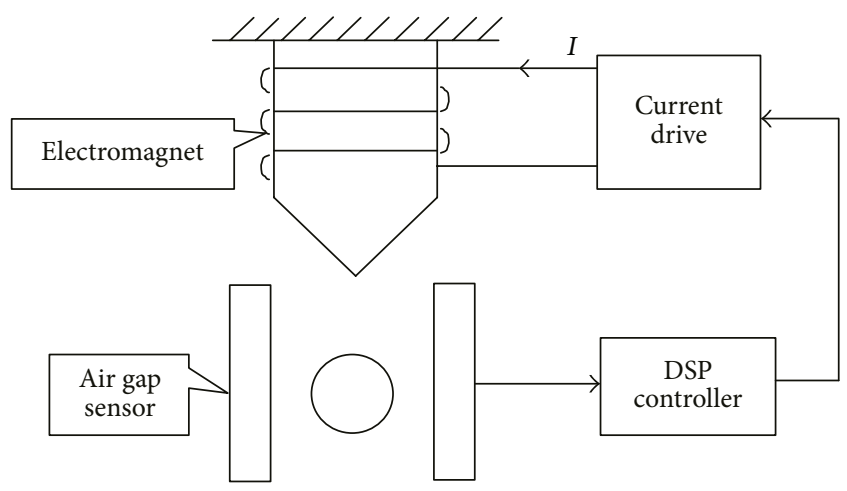

FIGURE 1: Single degree of freedom EMS.

The corresponding Routh table can be computed as in Table 1 .

It is easy to know according to Routh table that when $R^{\prime} \sqrt{m g / k}<K_{P}<\left(R^{\prime} s_{0} / 2 k\right) K_{D}+R^{\prime} \sqrt{m g / k}$, the system (2) is stable at the equilibrium point.

Furthermore, there is a bifurcation when $c_{4,1}=0$ or $c_{3,1}=0$. In fact, $K_{P}>R^{\prime} \sqrt{m g / k}$, so $c_{4,1}=0$ is impossible. This means that there is a bifurcation in the system only when $c_{3,1}=0$. Next, we will show the bifurcation is HOPF bifurcation.

When $c_{3,1}=0$, there is a pair of pure imaginary roots and a negative real root. The pure imaginary roots are [14]

$$
\lambda=\alpha\left(K_{P}, K_{D}\right) \pm \omega\left(K_{P}, K_{D}\right) j,
$$

where $\alpha\left(K_{P}, K_{D}\right)=0$ and $\omega\left(K_{P}, K_{D}\right)=\sqrt{K_{D} \sqrt{g / m k}}$.

The other root can be achieved through computation [14]:

$$
\alpha^{\prime}\left(K_{P}, K_{D}\right)=\frac{E s_{0}}{2 g}\left(\frac{R^{\prime}}{K_{P}}-1\right),
$$

where $E=\left[R^{\prime 2}\left(1+\omega^{2}\right)+K_{P}^{2}\right]^{-1}$.

In practice, $K_{P} \gg R^{\prime}$; then $\alpha^{\prime}\left(K_{P}, K_{D}\right)<0$.

So there is a HOPF bifurcation in EMS when $c_{3,1}=0$. That is to say, the system has periodical solutions which means that there is a self-excited vibration at this point.

We define the scope of $K_{P}$ in which the system is stable as

$$
W=\left(K_{P L}, K_{P R}\right),
$$

where $K_{P L}$ is the left pole of proportional feedback coefficient and $K_{P R}$ is the right pole, and

$$
\begin{gathered}
K_{P L}=R^{\prime} \sqrt{\frac{m g}{k}}, \\
K_{P R}=R^{\prime} \sqrt{\frac{m g}{k}}+\frac{R^{\prime} s_{0}}{2 k} K_{D} .
\end{gathered}
$$

\section{Parameters Design of Adaptive Controller}

The design of adaptive controller parameters is to choose the proper levitation air gap coefficient $K_{P}$ and the differential feedback coefficient $K_{D}$, so that they satisfy the following conditions: 
TABLE 1: Routh table of EMS Jacobian matrix.

\begin{tabular}{lcc}
\hline$c^{3}$ & $c_{1,1}=1$ & $c_{1,2}=K_{D} \sqrt{\frac{g}{k m}}$ \\
$\lambda^{2}$ & $c_{2,2}=K_{P} \sqrt{\frac{g}{k m}}-\frac{g}{k} R$ \\
$\lambda^{1}$ & $c_{3,1}=\frac{2}{s_{0}} \sqrt{\frac{m g}{k}}\left(\sqrt{\frac{m g}{k}}+\frac{K_{D} s_{0}}{2 k}-\frac{K_{P}}{R}\right)$ & \\
$\lambda^{0}$ & $c_{4,1}=K_{P} \sqrt{\frac{g}{k m}}-\frac{g}{k} R$ & \\
\hline
\end{tabular}

(1) $K_{P} \in W$;

(2) $K_{P}$ and $K_{P R}$ keep a certain far distance.

Condition (1) guarantees the stability. Condition (2) guarantees that the system has no HOPF bifurcation.

In EMS, the mass levitated is not always fixed. According to (8), (9), and (10), $W$ is a function of the mass levitated, and therefore it will slide with the mass varying. If $K_{P}$ keeps fixed, it will move beyond $W$ or approach to $K_{P R}$ while the mass levitated varying. The first condition broken will cause the system unstability, and the second condition broken will cause self-excited vibration. To avoid this, the proportional feedback coefficient should be adjusted with the varying of the mass levitated, confined in $W$, and kept far away from $K_{P R}$.

In order to provide enough stiffness and to avoid selfexcited vibration, in this paper, the proportional feedback coefficient is placed in the middle of interval $W$. That is, $K_{P}$ is determined by the following equation:

$$
K_{P}=K_{P L}+\frac{1}{2}\left(K_{P R}-K_{P L}\right)=R^{\prime} \sqrt{\frac{m g}{k}}+\frac{R^{\prime} s_{0}}{4 k} K_{D} .
$$

So it is necessary to know the levitated mass continuously and automatically; then we can adjust the proportional feedback coefficient so that both condition (1) and condition (2) can be satisfied together. But in fact, the levitated mass cannot be measured directly easily. To overcome this problem, a system variable must be found such out that it can reflect the variance of the levitated mass and can be real time measured.

As we know, when the system reaches the stable state, the current in the electromagnet, the electromagnet voltage, and the levitation mass satisfy the following equation:

$$
\begin{gathered}
I_{0}=s_{0} \sqrt{\frac{m g}{k}, \quad I_{0}}=\frac{U_{0}}{R}, \\
U=U_{0}+L \frac{d I}{d t},
\end{gathered}
$$

where $L=2 \mathrm{kN} / \mathrm{s}_{0}$ is the equivalent induction of electromagnet. Then, the following equation can be put forward:

$$
U=R s_{0} \sqrt{\frac{m g}{k}}+L \frac{d I}{d t} .
$$

In (13), the acceleration of gravity $g$ and the constant of electromagnet $k$ are fixed. The levitation air gap $s_{0}$,
TABLE 2: Parameters of Figure 2.

\begin{tabular}{lcccccc}
\hline$k$ & $m(\mathrm{~kg})$ & $R(\Omega)$ & $I_{0}(\mathrm{~A})$ & $K_{D}$ & $K_{I}$ & $s_{0}(\mathrm{~m})$ \\
\hline $7.5599 \times 10^{-4}$ & 750 & 2 & 34.3 & 200 & -20 & 0.01 \\
\hline
\end{tabular}

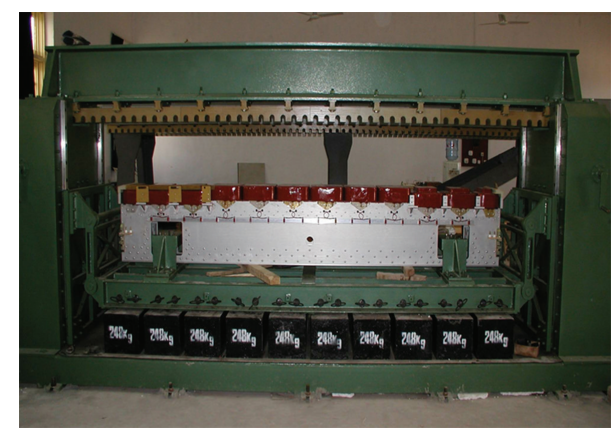

FIGURE 2: EMS experiment equipment of NUDT.

the resistance of electromagnet $R$, and the induction of electromagnet $L$ are also constant. The voltage of electromagnet $U$ and the current in the electromagnet $I$ can be measured by sensor. $d I / d t$, that is, the velocity of current changing, can be calculated by using the current $I$.

So the levitated mass can be calculated by the following equation:

$$
m=\left(\frac{U-L(d I / d t)}{R s_{0}}\right)^{2} \frac{k}{g}
$$

That is to say, we can get the variance of the levitated mass from the electromagnet voltage and current and get the variance scope of $W$ from the variance of the levitated mass. Then, the proportional feedback coefficient can be adjusted timely so that it can satisfy both condition (1) and condition (2) together.

\section{Simulation Testing}

We have an EMS experiment equipment as shown in Figure 2. Table 2 includes its parameters.

Then, according to (8), we have

$$
W=\left(\begin{array}{ll}
68632 & 97733
\end{array}\right)
$$

Simulation 1. Set $K_{P 0}$ in the middle of $W$; that is, $K_{P 0}=$ 83183; after the system runs for 40 seconds, the levitated mass decreases by $15 \%$ abruptly, and the step response of the system can be obtained as in Figure 3.

From Figure 3 we know that due to the abrupt decrease of the levitated mass at time $40 \mathrm{~s}$, the system, through a transitory regulating process, returns to a special state in which there is an evident self-excited vibration.

According to (8), (9), and (10), due to the decrease of the levitated mass, $W$ slides to 5356 . And because of the fixed $K_{P 0}$, the distance between $K_{P 0}$ and $K_{P R}$ decreases to 5356, which 


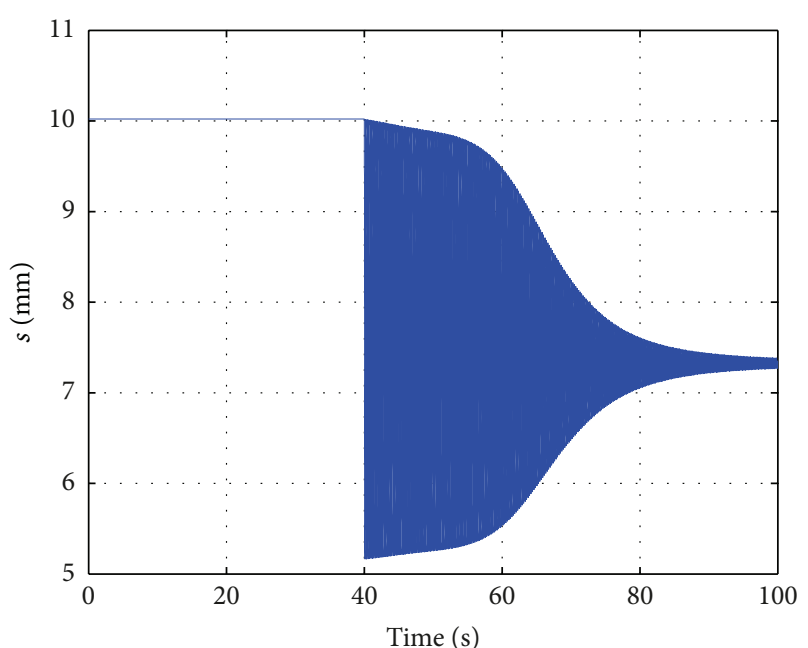

FIGURE 3: Step response of Simulation 1.

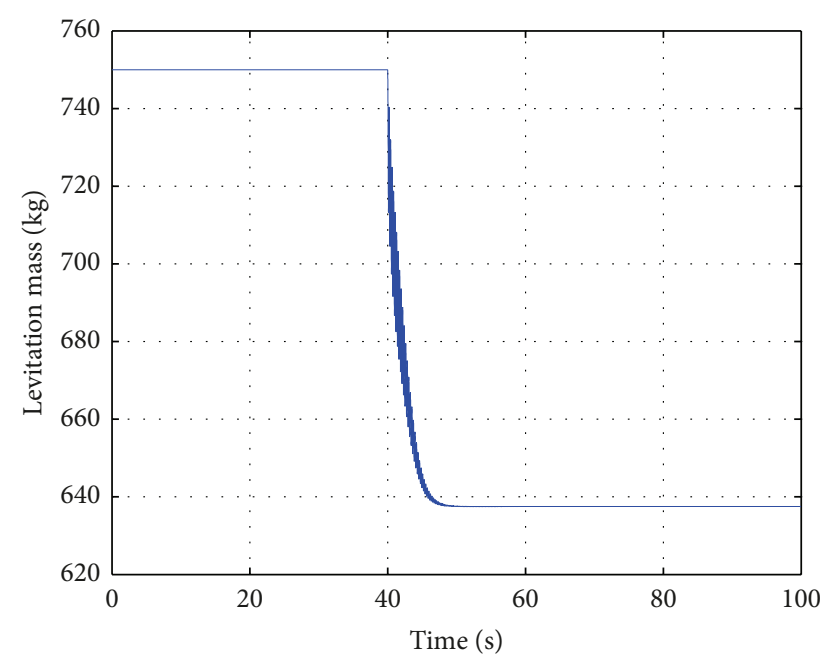

FIGURE 4: Curve of the estimated levitation mass.

results in $K_{P 0}$ approaching $K_{P R}$. Then, the system produces a self-excited vibration as shown in Figure 3.

Simulation 2. After the system runs for 40 seconds, the levitated mass decreases by $15 \%$ abruptly. The estimated levitation mass use (15) is shown in Figure 4 and the proportional feedback coefficient while the levitated mass varies according to HOPF bifurcation theory described in Section 4 is shown in Figure 5. The step response can be obtained as in Figure 6 in this case.

From Figure 6, we know that after the levitated mass abruptly decreases by $15 \%$, the system returns to the stable state, and there is no self-excited vibration.

According to the former computation, when the levitated mass decreases by $15 \%, W$ slides to left 5356. According to (12), the electromagnet voltage decreases by $7.8 \%$, and according to (8), $K_{P}$ slides to left 5356 . So there is no change of the relative distance between $K_{P}$ and $W$. Therefore, the dynamic characteristics of the system have not been changed.

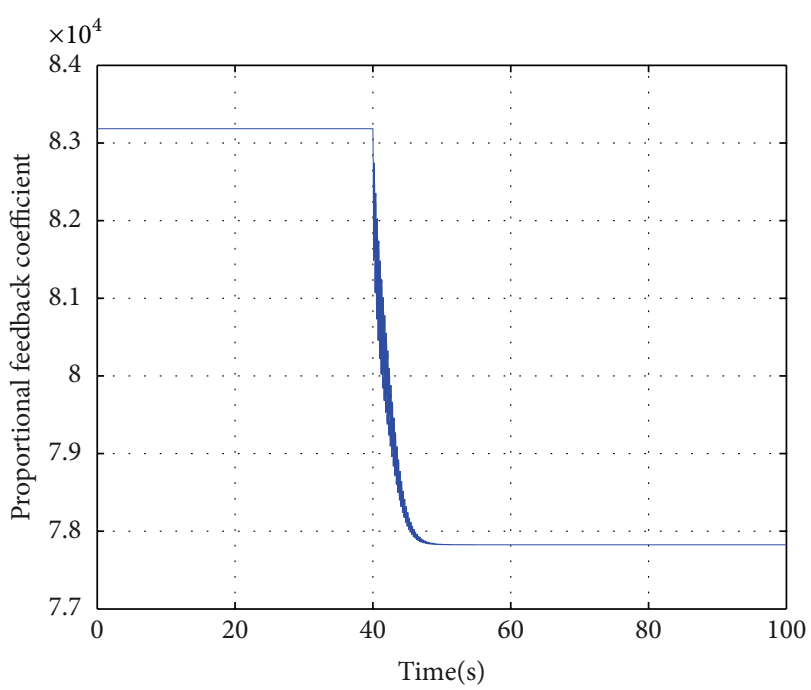

FIGURE 5: Curve of the proportional feedback coefficient.

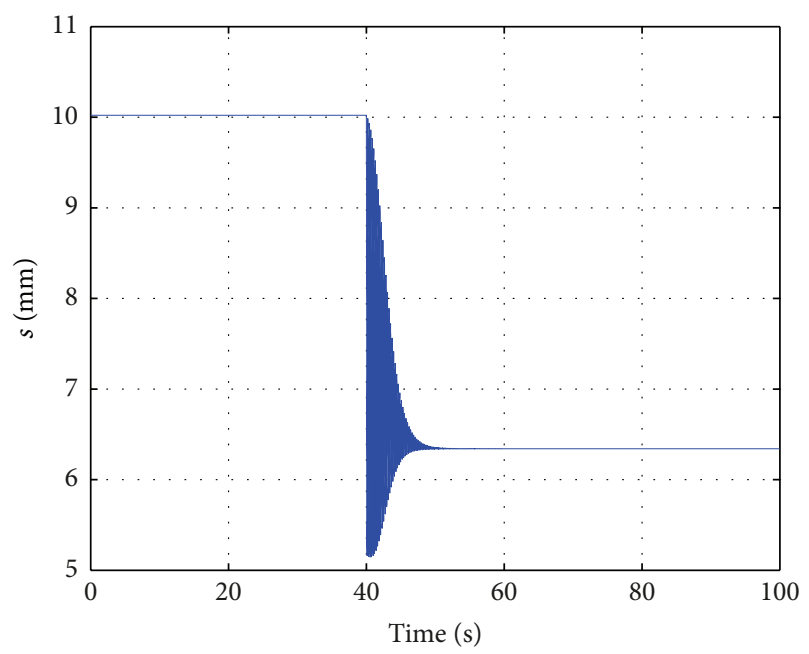

FIGURE 6: Step response of Simulation 2.

\section{Conclusion}

By analyzing the HOPF bifurcation of the nonlinear EMS type maglev system, a self-tuning adaptive control method is put forward, which uses an additional constraint as the adaptive rule. This constraint makes the controller parameter always far away from the HOPF bifurcation points while the mass levitated varies. Proved by simulation, adaptive control method in this paper can make electromagnetic suspension system still stable after an abrupt changing of the mass levitated and avoid its self-excited vibration.

The stability of the adaptive controller needs to be proven theoretically furthermore. And the adaptive relationship needs to be formalized furthermore.

\section{References}

[1] X. Wu, "Achievements of Shanghai Maglev demonstation operation line and the Maglev development strategy," in Proceedings of the Maglev, vol. 1, pp. 13-16, 2004. 
[2] X. Wu, Maglev Train, Shanghai Science and Technology Publishing Company, Shanghai, China, 2003.

[3] P. K. Sinha, Electromagnetic Suspension: Dynamics \& Control, Peter Peregrinus, London, UK, 1987.

[4] Y. Li and W. Chang, "Cascade control of an EMS Maglev vehicle's levitation control system," Acta Automatica Sinica, vol. 25, no. 2, pp. 247-251, 1999.

[5] L. She, "A research on magnetic suspension and balance systems' control problem," Experiments and Measurements in Fluid Mechanics, vol. 11, no. 4, pp. 51-56, 1997.

[6] P. K. Sinha and A. N. Pechev, "Model reference adaptive control of a maglev system with stable maximum descent criterion," Automatica, vol. 35, no. 8, pp. 1457-1465, 1999.

[7] Y. C. Kim and K. H. Kim, "Gain scheduled control of magnetic suspension system," in Proceedings of the American Control Conference, vol. 3, pp. 3127-3131, July 1994.

[8] J. Kaloust, C. Ham, J. Siehling, E. Jongekryg, and Q. Han, "Nonlinear robust control design for levitation and propulsion of a Maglev system," Control Theory and Applications, vol. 151, no. 4, pp. 460-464, 2004.

[9] A. J. van der Schaft, "L2-gain analysis of nonlinear systems and nonlinear state-feedback Ho control," IEEE Transactions on Automatic Control, vol. 37, no. 6, pp. 770-784, 1992.

[10] M. Fujita, T. Namerikawa, F. Matsumura, and K. Uchida, " $\mu$-synthesis of an electromagnetic suspension system," IEEE Transactions on Automatic Control, vol. 40, no. 3, pp. 530-536, 1995.

[11] D. L. Trumper, S. M. Olson, and P. K. Subrahmanyan, "Linearizing control of magnetic suspension systems," IEEE Transactions on Control Systems Technology, vol. 5, no. 4, pp. 427-438, 1997.

[12] B. D. Hassard, N. D. Kazarinoff, and Y.-H. Wan, Theory and Applications of Hopf Bifurcation, Cambridge University Press, Cambridge, UK, 1981.

[13] G. Liu and L. She, "HOPF bifurcation and vibration analysis of magnetic levitation system," Journal of Vibration, Measurement \& Diagnosis, vol. 23, no. 4, pp. 276-278, 2003.

[14] G. Liu, Vibration analysis of single-fulcrum magnetic levitation system [M.S. thesis], National University of Defense Technology, Changsha, China, 2003. 


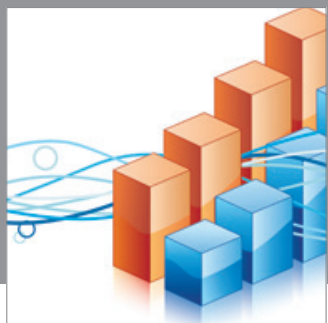

Advances in

Operations Research

mansans

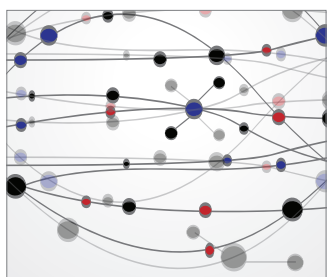

The Scientific World Journal
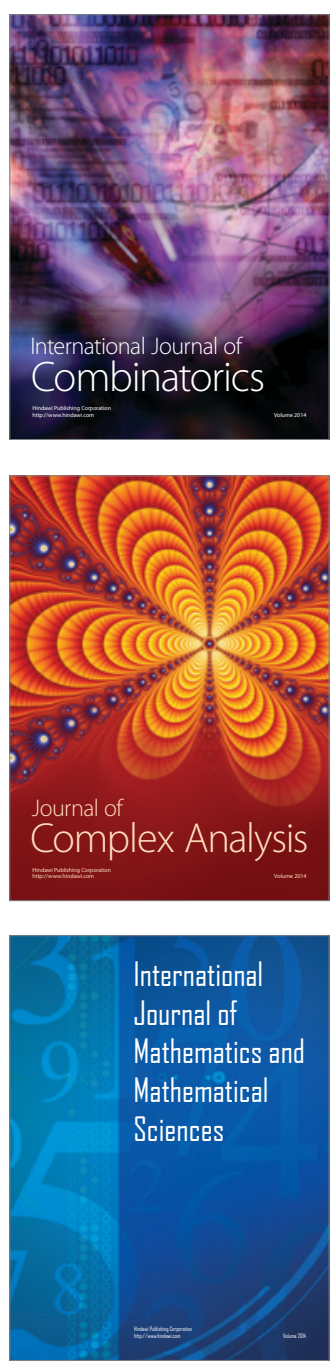
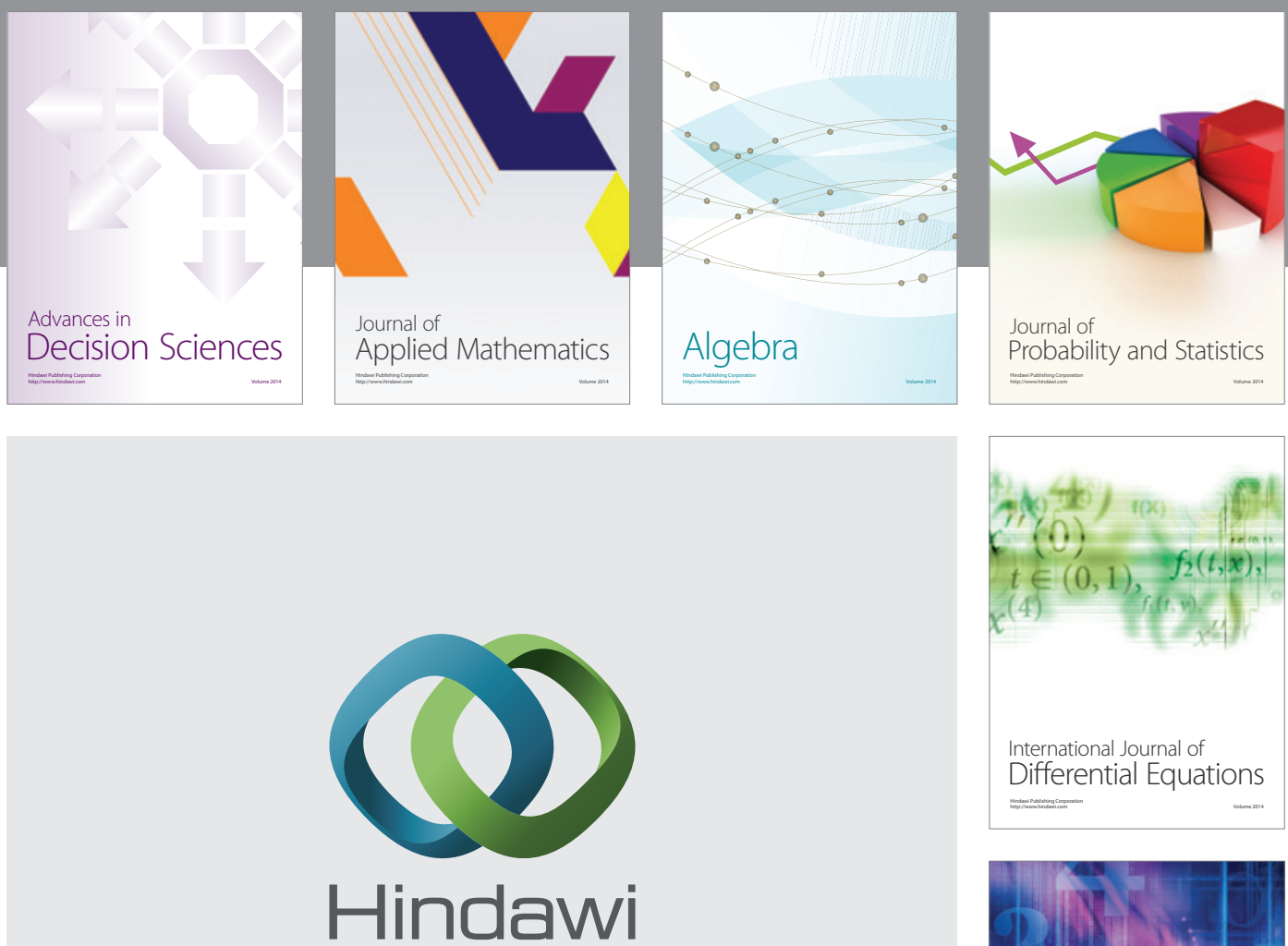

Submit your manuscripts at http://www.hindawi.com
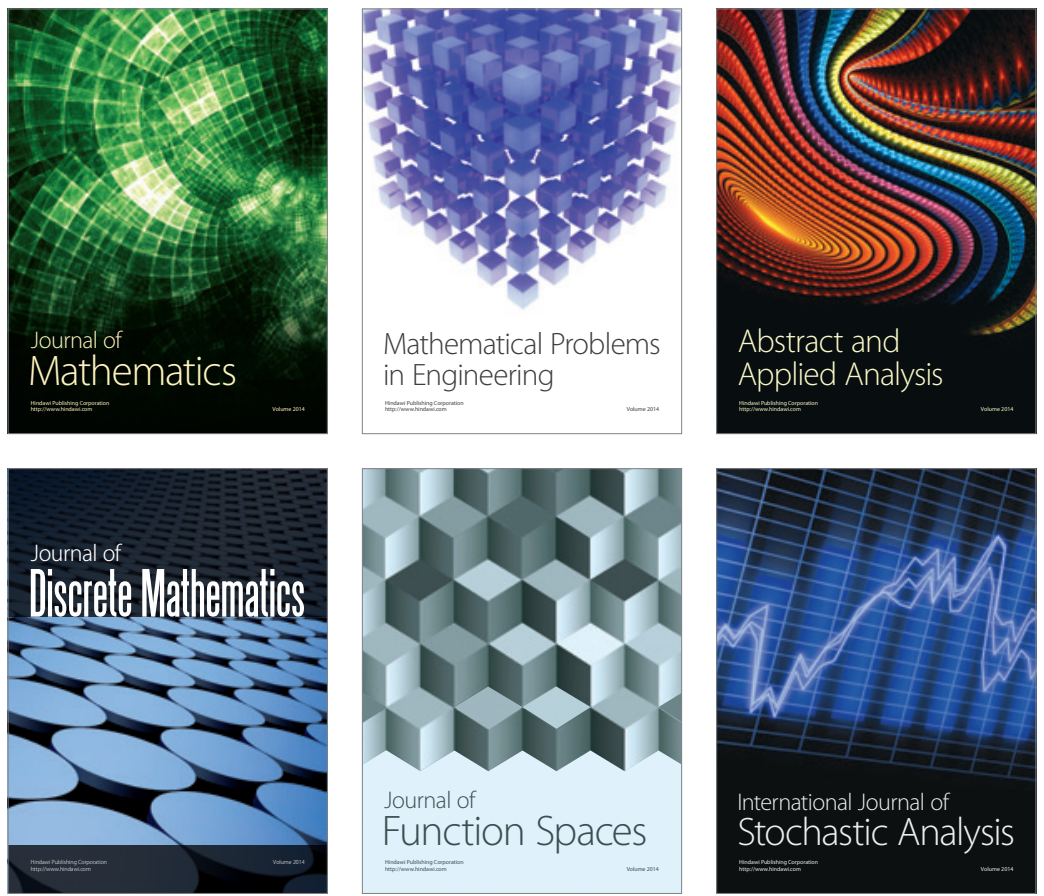

Journal of

Function Spaces

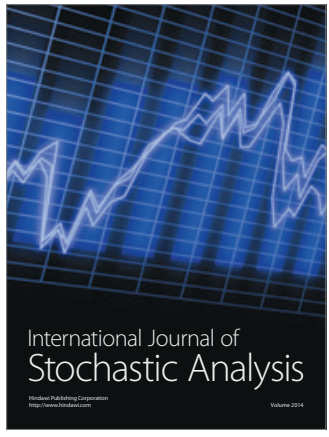

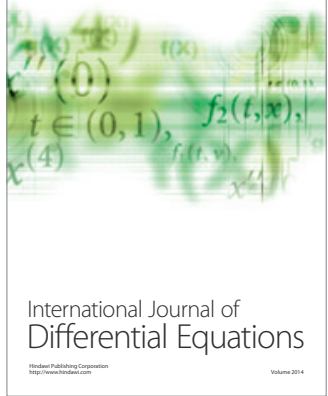
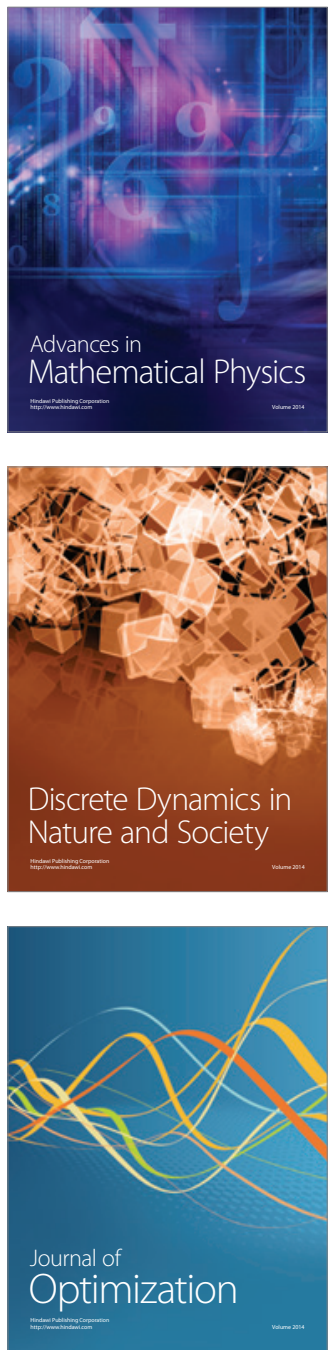